\title{
Hypertension in type 2 diabetes mellitus: associated treatment and degree of control
}

Fernández-García, José Carlos (1), Cortés-Salazar, Carmen María (2)

1. Endocrinology Department. Virgen de la Victoria University Hospital. Málaga (Spain).

2. Badolatosa Primary Care Centre. Seville (Spain).

\section{Objectives}

To evaluate the prevalence of hypertension in patients with type 2 diabetes mellitus (T2DM) and to analyze the clinical characteristics, the degree of achieved control and the associated antihypertensive treatment.

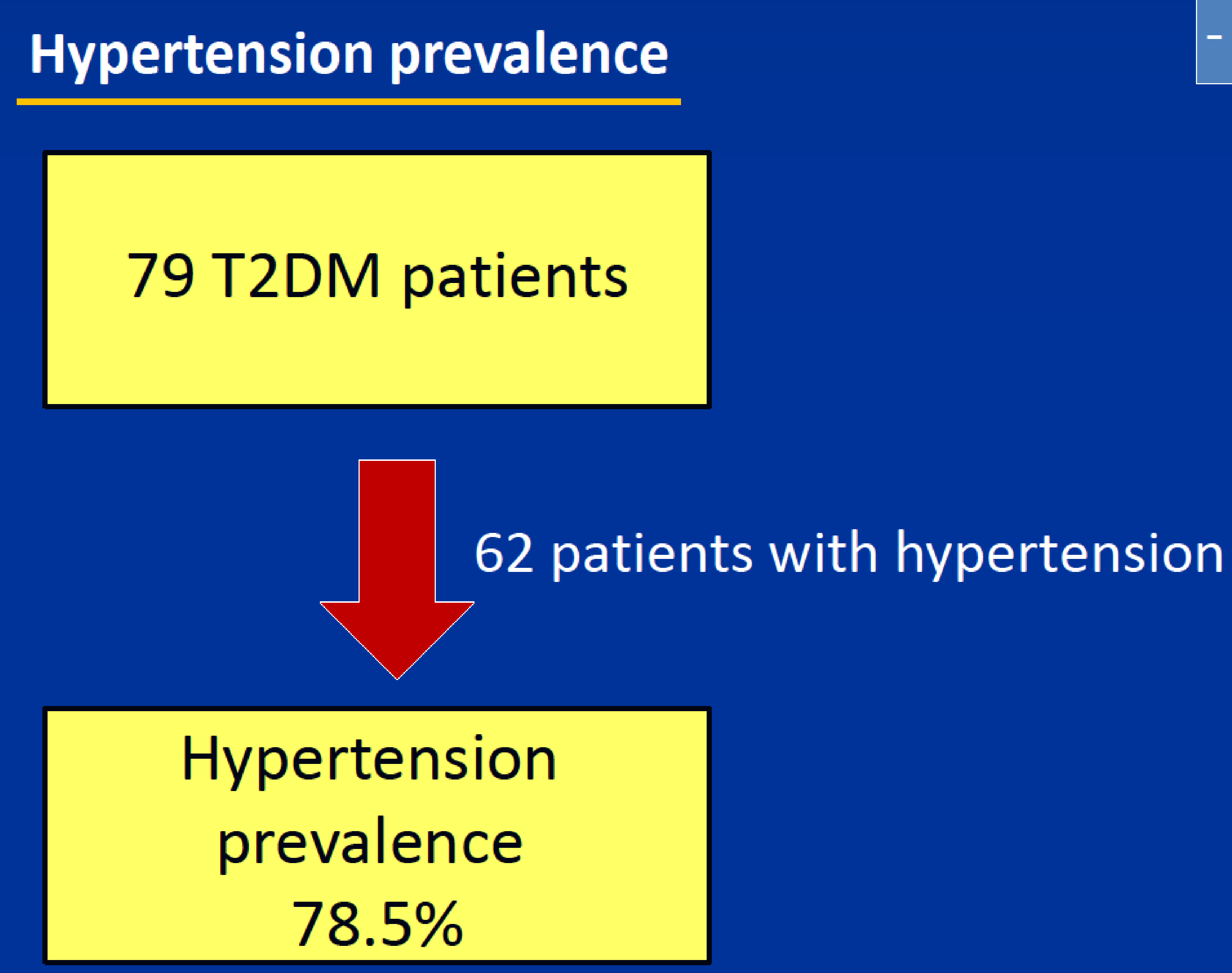

\section{Methods}

- Cross-sectional study

- Inclusion criteria: T2DM patient followed in a primary care setting.

- Data about age, sex, associated antihypertensive treatment, systolic blood pressure (SBP) and diastolic blood pressure (DBP) was collected

Statistical analysis was performed with SPSS v 15.0 for Windows.

Clinical characteristics of hypertensive patients

\begin{tabular}{|l|c|}
\hline Age (years) & $72.5 \pm 10.1$ \\
\hline Males $(\%)$ & 62.9 \\
\hline BMI $\left(\mathrm{kg} / \mathrm{m}^{2}\right)$ & $32,4 \pm 5.8$ \\
Hba1c $(\%)$ & $7,3 \pm 0,9$ \\
Mean SBP $(\mathrm{mmHg})$ & $126.5 \pm 13.7$ \\
Mean DBP $(\mathrm{mmHg})$ & $72.9 \pm 8.6$ \\
BP $<140 / 90 \mathrm{mmHg}(\%)$ & 77.6 \\
\hline
\end{tabular}

\section{Associated antihypertensive treatment (\%)}

\begin{tabular}{|c|c|}
\hline Diuretics & 61.3 \\
\hline Calcium Antagonists & 30.6 \\
ACE inhibitors & 35.5 \\
ARBs & 45.2 \\
\hline Average $(\mathrm{n})$ & 2.15 \\
\hline
\end{tabular}

\section{Distribution of antihypertensive therapy (\%)}

\section{Conclusions}

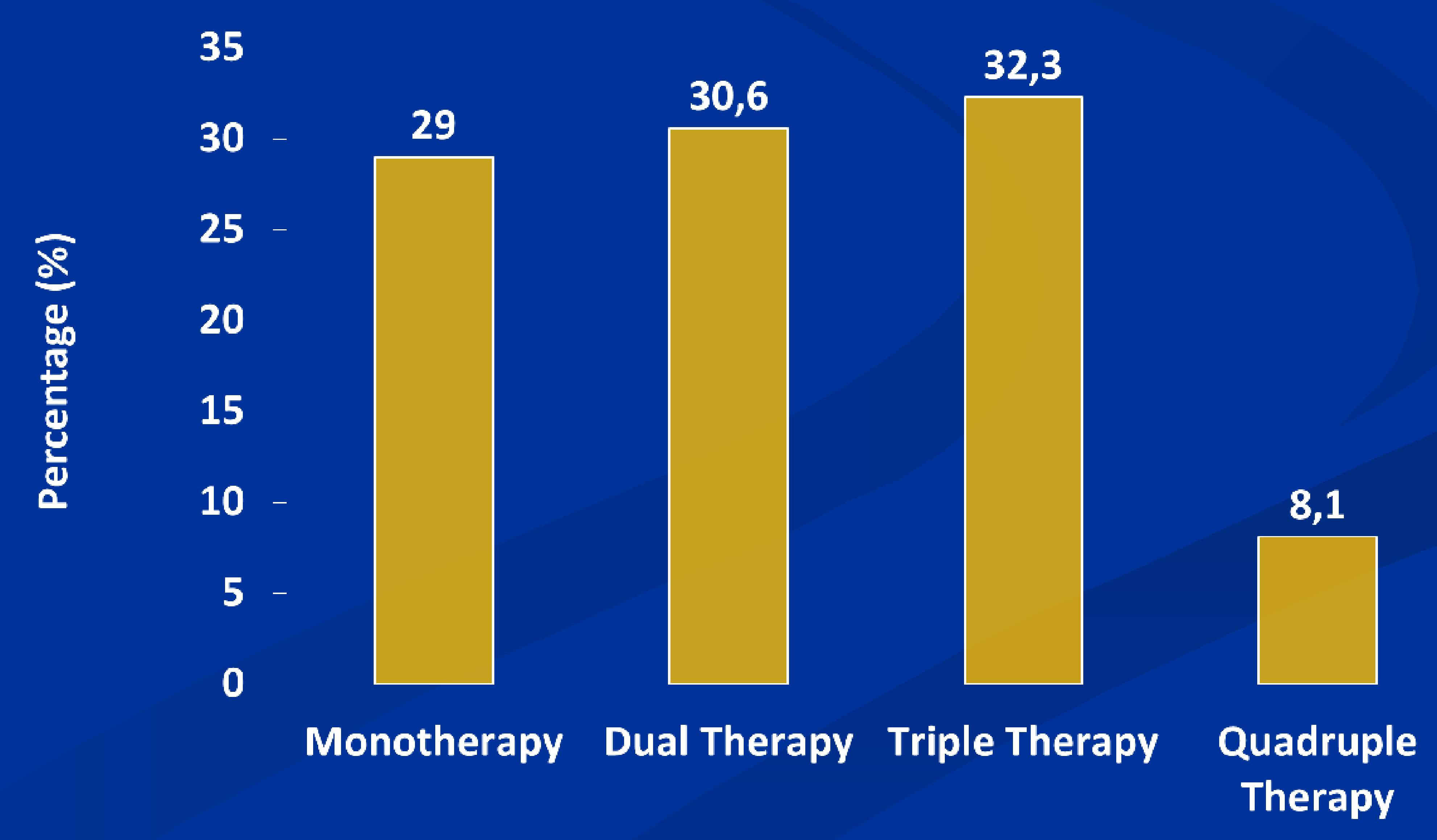

$\Rightarrow$ The vast majority of patients with T2DM have hypertension, but it is usually well-controlled.

$\Rightarrow$ At least two antihypertensive drugs were used is most patients to control blood pressure, being the most used drug class ARBs. 